\title{
Dynamic properties of structures with dampers modelled using fractional order derivatives
}

\author{
Zdzisław Pawlak, Roman Lewandowski \\ Institute of Structural Engineering \\ Poznan University of Technology \\ 60-965 Poznań, Poland \\ zdzislaw.pawlak@put.poznan.pl
}

\begin{abstract}
The focus of this paper is on determination of the dynamic parameters of structural systems with viscoelastic (VE) dampers described by Maxwell rheological models. Such parameters could be obtained after solving the appropriately defined nonlinear eigenvalue problem for frames with VE dampers. The solution to the nonlinear eigenvalue problem is obtained by equating to zero the determinant of the considered system of equations. Apart from complex conjugate eigenvalues, the real ones occurred when dampers that are described by the classic Maxwell model, are also determined.
\end{abstract}

Keywords: viscoelastic damper; rheological model; fractional derivative; nonlinear eigenvalue problem; dynamic properties;

\section{Introduction}

In civil engineering passive damping systems are mounted on structures in order to reduce excessive vibrations caused by winds and earthquakes [1-3]. Different kinds of mechanical devices, such as viscous dampers, viscoelastic dampers, tuned mass dampers, or base isolation systems, are used in the passive systems. In the past, several rheological models were proposed for describing the dynamic behaviour of VE dampers and materials [1-3]. In recent years, the fractional calculus has received considerable attention and has been used in modelling the rheological behaviour of VE materials and dampers [4, 5]. The fractional models have an ability to correctly describe the behaviour of VE materials and dampers using a small number of model parameters. However, in this case, the VE damper equation of motion is a fractional differential equation [6]. It is the aim of this paper to find the dynamic properties (i.e., natural frequencies and non-dimensional damping factors) for structures with VE dampers. The above-mentioned properties are defined on the basis of eigenvalues, obtained from the nonlinear eigenproblem. The approach, as presented in this work, differs from the standard one which mostly uses the state-space variables and the dynamic parameters are derived from the linear eigenproblem [7] or the non-linear eigenproblem [8], depending on the assumed model of damper. One of the most important achievements of the proposed formulation is the dimension of the problem, which is much smaller, compared with the standard approach. The solution to the nonlinear eigenvalue problem is obtained by equating to zero the determinant of the considered system of equations. The results of sample numerical calculations are presented and discussed. It is shown that the results of nonlinear eigenproblem which correspond to the classic models differ qualitatively from the results obtained for the fractional model.

\section{Rheological Model of Damper}

a)

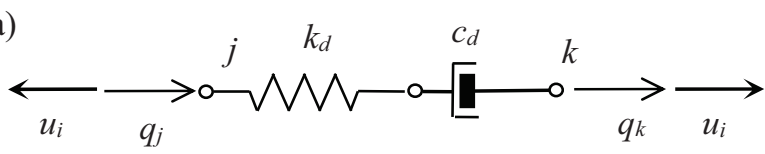

b)

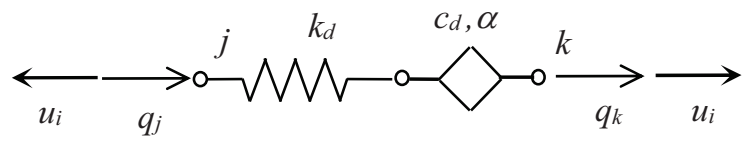

c)

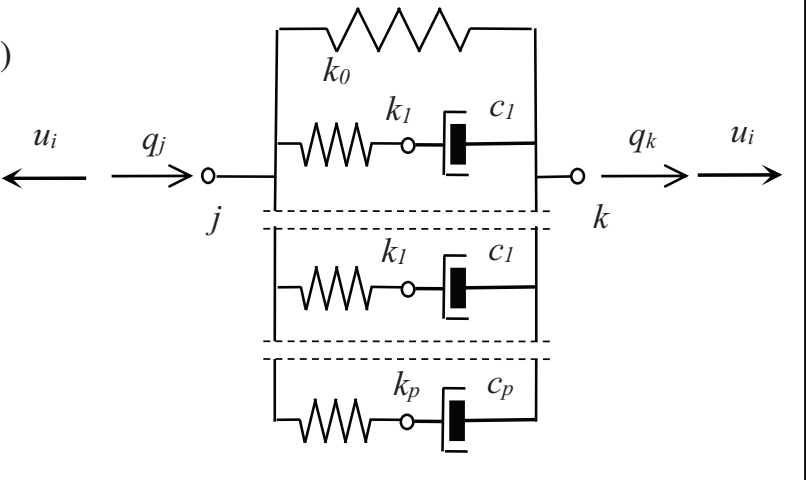

The rheological properties of VE dampers were described using three different Maxwell models, i.e., classic model (Fig. 1a), fractional model (Fig. 1b) and generalized model (Fig. 1c). The classic Maxwell model consists of a dashpot with the constant $c_{d}$, connected in series with a spring of the stiffness $k_{d}$.

\section{Figure 1. Rheological models of damper.}

In the case of the fractional Maxwell model of damper, instead of the dashpot we have a fractional dashpot (see Fig. 1b) with the constants: $c_{d}$ and $\alpha \quad(0<\alpha \leq 1)$, which denotes the order of fractional derivative [6].

In the generalized Maxwell model (Fig. 1c), there is an additional element of the stiffness $k_{0}$, which is connected in parallel with the other elements of the system, described 
respectively by stiffness $k_{l}$ and damping $c_{l},(l=1,2, \ldots, p)$. The equations of motion for the classic or fractional and generalized Maxwell models could be written as follows:

$$
\begin{aligned}
& D_{t}^{\alpha} u_{i}(t)+v u_{i}(t)=k_{d} D_{t}^{\alpha} \Delta q_{i}(t) \\
& v_{l} u_{l i}(t)+D_{t}^{1} u_{l i}(t)=k_{l} D_{t}^{1} \Delta q_{i}(t), \\
& u_{0 i}(t)=k_{0} \Delta q_{i}(t) \quad l=1,2, \ldots, p
\end{aligned}
$$

where, $\Delta q_{i}(t)=q_{j}-q_{k}, v=k_{d} / c_{d}, v_{l}=k_{l} / c_{l}$. Moreover, $u_{i}, u_{l i}$ and $q_{j}, q_{k}$ denote the dampers force, the force in the $j$-th Maxwell unit and nodal displacements, respectively. The symbol $D_{t}^{\alpha}(\bullet)$ denotes the Riemann-Liouville fractional derivative of the order $\alpha$ with respect to time, $t$. More information concerning the fractional derivative can be found in [6]. For consistent notation, we introduce $D_{t}^{1}(q)=\&(t)$. The equation of motion for the classic Maxwell model could be obtained after introducing into (1) $\alpha=1$.

\section{Structural system with dampers}

\section{A. The equation of motion}

In this paper, the structure with VE dampers is treated as an elastic linear system modelled as a shear frame. The mass of the system is lumped at the level of storeys. Viscoelastic dampers are installed between two successive storeys. The equation of motion of the structure with dampers can be written as follows:

$$
\mathbf{M}_{s} \&_{s}(t)+\mathbf{C}_{s} \mathbf{q}_{s}(t)+\mathbf{K}_{s} \mathbf{q}_{s}(t)=\mathbf{f}(t)+\mathbf{p}(t)
$$

where the symbols $\mathbf{M}_{s}, \mathbf{C}_{s}$ and $\mathbf{K}_{s}$ denote the mass, the damping, and the stiffness matrices, respectively. Moreover, $\mathbf{q}_{s}(t)=\left[q_{s, 1}, \ldots, q_{s, j}, \ldots, q_{s, n}\right]^{T} \quad$ denotes the vector of displacements of the structure and $\mathbf{p}(t)=\left[p_{1}, \ldots, p_{j}, \ldots, p_{n}\right]^{T}$ the vector of excitation forces. The components of vector $\mathbf{f}(t)=\left[f_{1}, f_{2}, \ldots, f_{n}\right]^{T}$ are the interaction forces between the frame and the dampers (Fig.. 2).

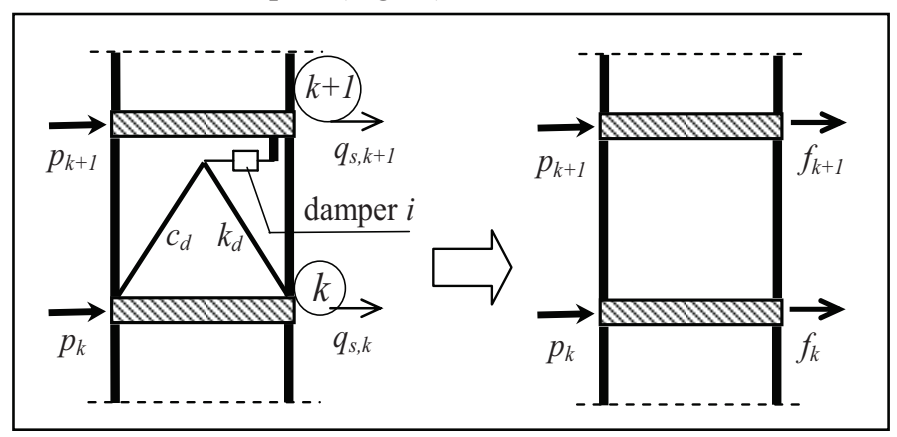

Figure 2. Diagram of frame and the interaction forces.

If a structure with only one damper denoted as the damper number $i$, mounted between two successive storeys, $k$ and $k+1$, is considered, then the vector of damper forces could be written in the following form:

$$
\mathbf{f}_{i}(t)=\left[0, \ldots, f_{k}=u_{i}, f_{k+1}=-u_{i}, \ldots . ., 0\right]^{T}=\mathbf{e}_{i} u_{i}(t)
$$

where, $\mathbf{e}_{i}=\left[0, \ldots, e_{k}=1, e_{k+1}=-1, \ldots, 0\right]^{T}$. For a structure with $m$ dampers the vector of interaction forces is the sum of vectors $\mathbf{f}_{i}(t)$, i.e.:

$$
\mathbf{f}(t)=\sum_{i=1}^{m} \mathbf{f}_{i}(t) .
$$

\section{B. The Laplace transform}

After applying the Laplace transform and taking into account that:

$$
\mathcal{L}[\mathbf{q}(t)]=\overline{\mathbf{q}}, \mathcal{L}\left[D_{t}^{\alpha} \mathbf{q}(t)\right]=s^{\alpha} \overline{\mathbf{q}}, \mathcal{L}\left[D_{t}^{1} \mathbf{q}(t)\right]=s \overline{\mathbf{q}},
$$

the equation of motion (2) can be written as:

$$
\left(s^{2} \mathbf{M}_{s}+s \mathbf{C}_{s}+\mathbf{K}_{s}\right) \overline{\mathbf{q}}_{s}(s)=\overline{\mathbf{f}}(s)+\overline{\mathbf{p}}(s) .
$$

The vectors $\overline{\mathbf{q}}_{s}(s), \overline{\mathbf{f}}(t)$ and $\overline{\mathbf{p}}(s)$ denote the Laplace transforms of displacements and forces, respectively. According to (3), for damper $i$, the force transform is $\overline{\mathbf{f}}_{i}(s)=\mathbf{e}_{i} \bar{u}_{i}(s)$. The Laplace transform converts (1) into one relationship which is valid for each considered model of damper:

$$
\bar{u}_{i}(s)=\left[k_{v i}+G_{i}(s)\right] \Delta \bar{q}_{i}(s) .
$$

The quantities $k_{v i}$ and $G_{i}(s)$ are defined as:

$$
\begin{array}{ll}
k_{v i}=0 & G_{i}(s)=\frac{s^{\alpha_{i}} \cdot k_{d i}}{v_{i}+s^{\alpha_{i}}} \\
k_{v i}=k_{0 i} & G_{i}(s)=\sum_{l=1}^{p} \frac{s \cdot k_{l i}}{v_{l i}+s}
\end{array}
$$

for classic $\left(\alpha_{i}=1\right)$ or fractional models and for generalized Maxwell model, respectively. Now the second index in the symbols $k_{0 i}, k_{l i}, v_{l i}$ refers to the damper's number. Moreover, $\Delta \bar{q}_{i}(s)=-\mathbf{e}_{i}^{T} \overline{\mathbf{q}}_{s}(s)$.

Finally, one may rewrite (6) in the following form:

$$
\left[s^{2} \mathbf{M}_{s}+s \mathbf{C}_{s}+\mathbf{K}+\mathbf{G}(s)\right] \overline{\mathbf{q}}_{s}(s)=\overline{\mathbf{p}}(s),
$$



where, $\mathbf{K}=\mathbf{K}_{s}+\mathbf{K}_{v}, \mathbf{K}_{v}=\sum_{i=1}^{m} k_{v i} \mathbf{e}_{i} \mathbf{e}_{i}^{T}, \mathbf{G}(s)=\sum_{i=1}^{m} G_{i}(s) \mathbf{e}_{i} \mathbf{e}_{i}^{T}$,
$m$ stands for the number of dampers.

\section{Nonlinear eigenproblem}

For $\overline{\mathbf{p}}(s)=\mathbf{0}$, the equation of motion (9) expresses a nonlinear eigenproblem from which the eigenvalues and eigenvectors can be determined [8]. In the case of the fractional Maxwell model it is possible to write the relationship:

$$
\left[\left(s^{2} \mathbf{M}_{s}+s \mathbf{C}_{s}+\mathbf{K}\right)+\sum_{i=1}^{m} \frac{s^{\alpha_{i}}}{\left(v_{i}+s^{\alpha_{i}}\right)} \mathbf{B}_{i}\right] \overline{\mathbf{q}}_{s}=\mathbf{0},
$$

where, $\mathbf{B}_{i}=k_{i} \mathbf{e}_{i} \mathbf{e}_{i}^{T}$. For the classic Maxwell model $\alpha_{i}=1=$

The solution to the system (10) yields a set of complex and conjugate eigenvalues. The number of pairs of eigenvalues equals the number of degree of freedom for the considered system. Moreover, for a structural system with the classic or the generalized Maxwell models of dampers we obtain a set of real eigenvalues of which the number equals the number of all dashpots occurring in the damper models. The calculation carried out by the authors suggests that for the dampers described by the fractional Maxwell model, real solutions do not exist.

A nonlinear eigenproblem can be solved using the continuation method which is similar to the one described in the paper [8]. Another possibility to obtain the values $s_{i}$ is a method of equating to zero the determinant of the system of equations [9]. It is to be noted that for $\alpha_{i}=1$ the value existing in the denominator in (10) leads to the singularity when $s=-v_{i}$. In order to eliminate these singularities, we transform the system of equations (10) into the following form:

$\left[\prod_{i=1}^{m}\left(v_{i}+s^{\alpha_{i}}\right)\left(s^{2} \mathbf{M}_{s}+s \mathbf{C}_{s}+\mathbf{K}\right)+\sum_{i=1}^{m} s^{\alpha_{i}} \prod_{\substack{k=1 \\ k \neq 1}}^{m}(11)\right.$

In this work, real and complex eigenvalues are obtained by searching the value of determinant of the system (11) and by evaluating the roots of determinant function.

\section{Dynamic properties of structure}

The dynamic behaviour of a frame with viscoelastic dampers is characterized by the natural frequencies $\omega_{i}$ and the non-dimensional damping parameters $\gamma_{i}$. Similarly to viscous damping, the above-mentioned properties are defined as follows:

$$
\omega_{i}^{2}=\mu_{i}^{2}+\eta_{i}^{2}, \quad \gamma_{i}=-\mu_{i} / \omega_{i}
$$

where $\mu_{i}=\operatorname{Re}\left(s_{i}\right), \eta_{i}=\operatorname{Im}\left(s_{i}\right)$. For the real eigenvalues $s_{i}$ relationships (12) are not valid. The real eigenvalues correspond to the rheological properties of the considered dampers.

\section{Results of Calculation}

In the numerical example, a two-storey building structure modelled as a shear plane frame with the rigid beams is considered. The mass is lumped and same at every floor: $m_{s}=2000 \mathrm{~kg}$. The bending rigidity of each storey is $k_{s}=4000 \mathrm{kN} / \mathrm{m}$. The viscoelastic damper with the stiffness parameter $k_{1}=2500 \mathrm{kN} / \mathrm{m}$ and damping $c_{1}=50 \mathrm{kNs}^{\alpha} / \mathrm{m}$ is mounted on the first storey. Thus, the coefficient $v_{1}=50$. On the second floor there is a damper, characterized by the parameters: $k_{2}=600 \mathrm{kN} / \mathrm{m}, c_{2}=30 \mathrm{kNs}^{\alpha} / \mathrm{m}, v_{2}=20$.

Firstly, the calculations were carried out for a frame with dampers described by the fractional Maxwell model, for which the value of the fractional parameter was $\alpha=0.6$. After equating to zero the determinant of the system of equations (11), we obtain the characteristic equation which enables four complex and conjugate eigenvalues $s_{i}$ to be derived (see Table I).

TABLE I. The EIgenValues - Fractional MaXwell Model

\begin{tabular}{|c|c|c|}
\hline Root number $i$ & $\mathfrak{R}\left(s_{i}\right)$ & $\mathfrak{I}\left(s_{i}\right)$ \\
\hline 1,2 & -0.7254 & \pm 28.3351 \\
3,4 & -1.8983 & \pm 74.8284 \\
\hline
\end{tabular}

The value determinant $\operatorname{det}(\mathrm{A})=Z(s)=a+i \cdot b \quad$ is $\quad$ a complex number which depends on the complex variable $s=x+i \cdot y$, where $i=\sqrt{-1}$. Thus, the value of the determinant equals zero only if its real and imaginary part

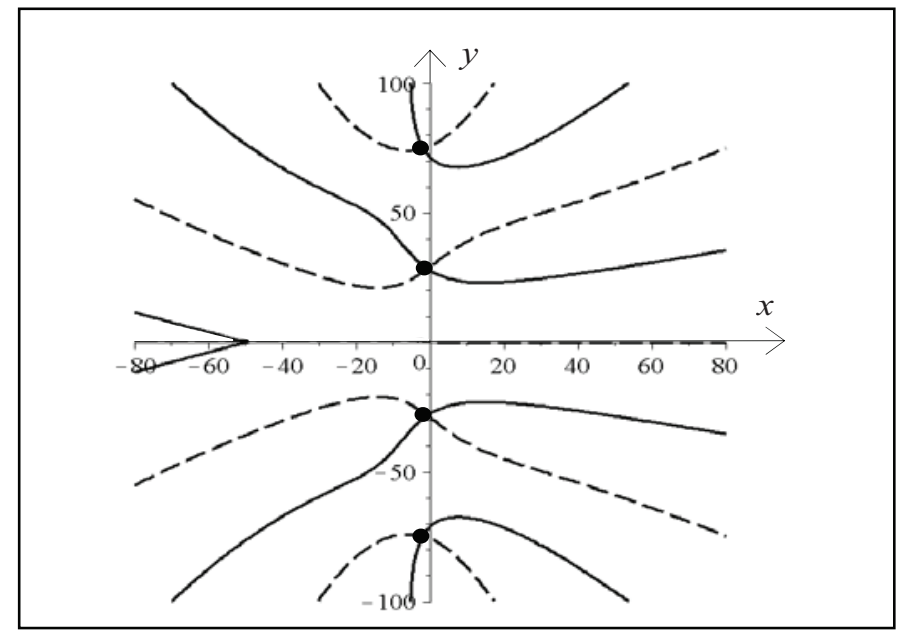

simultaneously is equal to zero, $a(x, y)=0$ and $b(x, y)=0$. The roots $s_{i}=x_{i}+i \cdot y_{i}$ of a characteristic equation are in a complex plane at the intersection of lines, along which the real 
part is equal to zero (solid line in Fig. 3) and the imaginary part is equal to zero (dashed line in Fig. 3).

Figure 3. Plot of functions $\mathfrak{R}(Z), \quad \mathfrak{I}(Z)$ - fractional Maxwell model.

In Fig. 3 one may observe four such intersection points of which the coordinates coincide with the values given in Table I.

Next, the dampers were modeled using the classic Maxwell model. The eigenproblem derived in the form of (11) was solved by equating the determinant of the system of equations to zero. This leads to a characteristic equation of which the solution yields four complex, conjugate eigenvalues $s_{i}$ and four real eigenvalues (see Table II).

TABLE II. The Eigenvalues - Classic MaXwell Model

\begin{tabular}{|c|c|c|}
\hline Root number $i$ & $\mathfrak{R}\left(s_{i}\right)$ & $\mathfrak{I}\left(s_{i}\right)$ \\
\hline 1,2 & -2.9931 & \pm 29.7644 \\
\hline 3,4 & -3.6167 & \pm 80.3574 \\
\hline 5 & -17.6578 & 0 \\
\hline 6 & -20 & 0 \\
\hline 7 & -39.1227 & 0 \\
\hline 8 & -50 & 0 \\
\hline
\end{tabular}

The roots of the number 6 and 8 correspond to the solutions $s_{i}=-v_{i}$, that means a singular solution of (10), which should not be treated as the eigenvalues. For these points, the value of the determinant, as derived from eigenproblem (10), tends to infinity.

The discussed solutions are presented in Fig. 4, as the points of intersection of the zero level lines of the surface $\mathfrak{R}(\mathrm{Z}(\mathrm{x}, \mathrm{y}))$ and surface $\mathfrak{I}(\mathrm{Z}(\mathrm{x}, \mathrm{y}))$ derived from $(11)$.

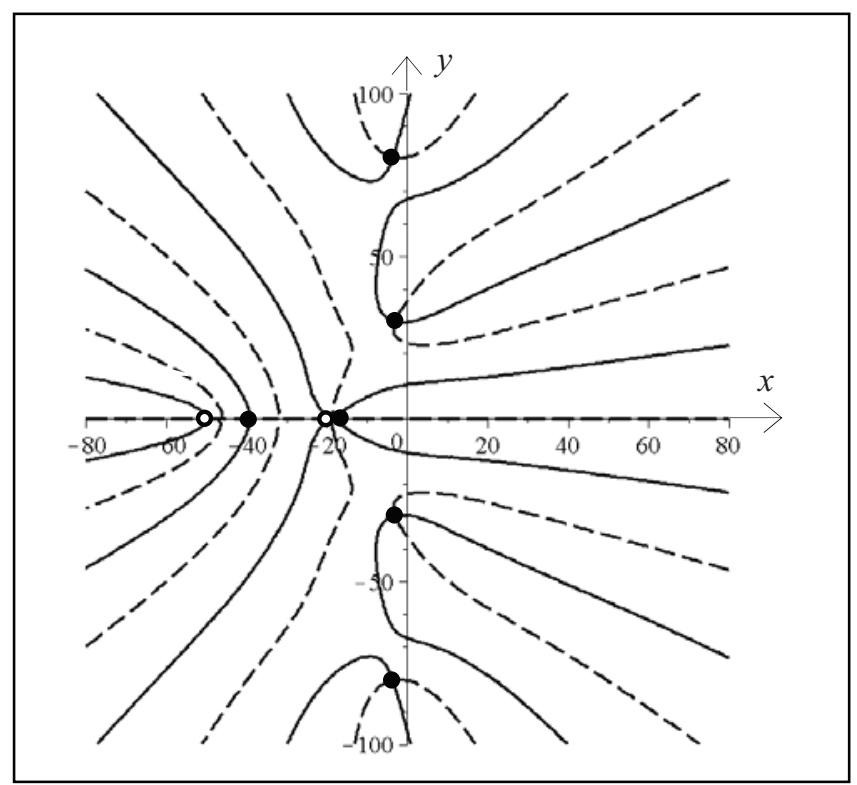

Figure 4. Plot of functions $\mathfrak{R}(Z)$ and $\mathfrak{I}(Z)$ - classic Maxwell model.
The real solutions $s_{5}$ and $s_{7}$ given in Table II coincide with the rheological properties of dampers mounted in structure.

\section{Concluding Remarks}

Comparing the results of calculations for a frame with dampers modeled using the classic Maxwell model and the results obtained for the fractional Maxwell model, we may observe qualitative differences. The solution to the nonlinear eigenproblem leads to a number of pairs of complex and conjugate eigenvalues $s_{i}=x_{i} \pm i \cdot y_{i}$. Moreover, in the case of the classic Maxwell model of damper we obtain some real eigenvalues for $x_{i}<0$ and $y_{i}=0$. For the fractional Maxwell model, real solutions do not exist because of discontinuity in the imaginary part of the determinant $\operatorname{det}(\mathrm{A})=Z(x, y) \quad$ (see Fig. 5).

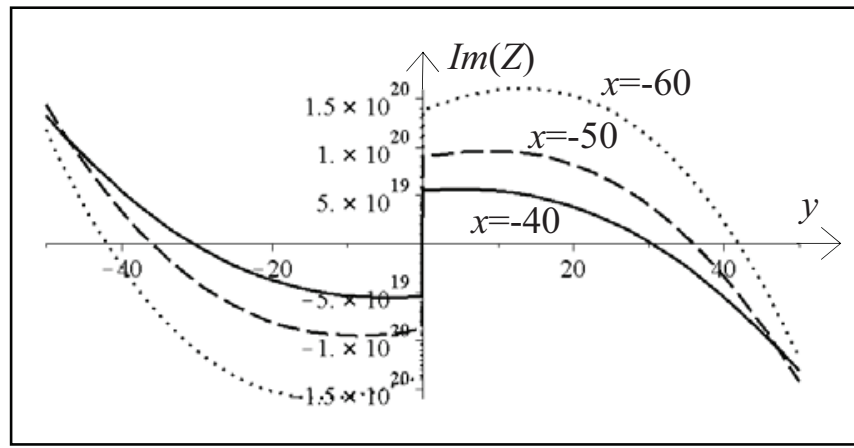

Figure 5. Diagram of imaginary part of determinant function.

\section{Acknowledgment}

The authors wish to acknowledge the financial support received from the Poznan University of Technology (Grant No. DS 11-088/12) in connection with this work.

\section{REFERENCES}

[1] S. W. Park, Analytical modelling of viscoelastic dampers for structural and vibration control. International Journal of Solids and Structures, 38, 8065-8092, 2001.

[2] C. Christopoulos, A. Filiatrault, Principles of Passive Supplemental Damping and Seismic Isolation. IUSS Press, Pavia, Italy, 2006.

[3] I. Takewaki, Building control with passive dampers, Optimal performance-based design for earthquakes. Wiley and Sons (Asia), Singapore, 2009.

[4] R. L. Bagley, P. J. Torvik, Fractional calculus - a different approach to the analysis of viscoelastically damped structures. AIAA Journal, 27, 1412-1417, 1998.

[5] T. Chang, M. P. Singh, Seismic analysis of structures with a fractional derivative model of viscoelastic dampers. Earthquake Engineering and Engineering Vibration, 1, 251-260, 2002.

[6] I. Podlubny, Fractional Differential Equations. Academic Press, 1999.

[7] R. Lewandowski, A. Bartkowiak, H. Maciejewski, Dynamic analysis of frames with viscoelastic dampers: a comparison of dampers models, Structural Engineering and Mechanics, 41, 113-137, 2012. 
[8] R. Lewandowski, Z. Pawlak, Dynamic Analysis of Frames with Viscoelastic Dampers Modelled by Rheological Models with Fractional Derivatives. Journal of Sound and Vibration, 330, 923-936, 2011.

[9] R. Lewandowski, Z. Pawlak, Description of dynamic behavior of frame structures with fractional viscoelastic dampers, 6th ICCSM International Congress of Croatian Society of Mechanics, September 30 - October 2, 2009, Dubrovnik, Croatia. 